\title{
¿Cómo pez en el agua? Aporías de la sociología disposicional 1
}

\author{
Like fish in the water? On the disjuncture of dispositional sociology
}

\author{
Claudio E. Benzecry* \\ * Profesor Asociado de Comunicación y Sociología - Northwestern University, Estados \\ Unidos | claudio.benzecry@northwestern.edu
}

Artículo traducido por Santiago García Martín

\section{PALABRAS CLAVE}

Pierre Bourdieu

Disposiciones

Incorporación

Sociología Pragmática

Sociología Psicológica

Sociología Relacional

\section{KEYWORDS}

Pierre Bourdieu

Disposition

Embodiment

Sociology of Conventions

Psychological Sociology

Relational Sociology

\section{RESUMEN}

Pierre Bourdieu acuña el concepto de habitus, para interrogar la articulación entre corporalidad, cognición, procesos de singularización y temporalización, y lo colectivo .Este trabajo problematiza las aporías que se siguen de semejante ambición semántica. El artículo continúa con una breve presentación de los diferentes usos de habitus en la obra de Bourdieu, seguido de una discusión acerca de cómo el concepto ha sido desplegado en la investigación por sociólogos estadounidenses. La tercera y principal sección se detiene en las aporías que devienen del uso extendido del concepto, y las distintas sendas críticas que han recorrido los especialistas del tema. Esta última sección se centra, no tanto en las críticas que la obra de Bourdieu ha recibido, sino en la tensión que la producción erudita presenta respecto de las explicaciones más disposicionalistas de la acción social. En este sentido presento seis discusiones que apuntan a ciertas conexiones conceptuales o semánticas de habitus que han sido dadas por sentadas, y que han sido retomadas luego por autores como Lahire, Steinmetz, Wacquant, Auyero, y Boltanski.

\begin{abstract}
Pierre Bourdieu coined the concept of habitus to point at the connection between embodiment, cognition, processes of singularization and temporalization, and the collective. This chapter discusses the aporias that result from this semantic ambition. The chapter starts with a brief presentation of the many uses of habitus in Bourdieu's own work; what follows after is how the concept has been deployed in research by US sociologists; the third and main section of the chapter looks at the aporias provoked by the concept's extension and the many critical avenues pursued by other scholars after it. This last section focuses less on criticisms to Bourdieu's oeuvre and more on scholarship produced in tension with dispositional accounts of social action. In it I present six conversations that point at conceptual or semantic connections that are taken for granted in habitus and opened up for sociological inquiry by scholars such as Lahire, Steinmetz, Wacquant, Auyero, Elias, and Boltanski.
\end{abstract}




\section{Introducción}

¿De qué modos se conectan cuerpo y biografía entre sí? ¿Cuáles son aquellas categorías que nos permiten expresar juicios basados en experiencias pasadas? ¿Cómo dar cuenta de las tensiones entre nuestro pasado y el potencial que alberga nuestro futuro? ¿De qué manera el contexto inmediato enmarca nuestra inmersión sensorial en el mundo? Y más aún, ¿qué significado tiene la relación entre las capacidades que nuestros cuerpos detentan y nuestras biografías? Para responder a estas preguntas Pierre Bourdieu acuña el concepto de habitus, focalizándose en la articulación entre corporalidad, cognición, procesos de singularización y temporalización, y lo colectivo. Con el objetivo de observar nuestras capacidades prácticas duraderas, o las circunstancias o condiciones bajo las cuales éstas pierden sentido, el sociólogo francés ocupó cuarenta años de su carrera desarrollando, ampliando y refinando en cuatro caminos diferentes aquello que explica la disposición a actuar.

La primera variante explicativa, desarrollada durante sus primeros estudios en Argelia, se concentraba en la disyunción que los agentes experimentaban entre las disposiciones individuales adquiridas y las estructuras objetivas del nuevo mundo al cual debían adaptarse. La segunda, comenzó luego de su trabajo sobre Erwin Panofsky, seguido más tarde por sus estudios sobre Heidegger y Flaubert, indagaciones centradas en la relación entre las disposiciones individuales y colectivas. La tercera, versión más proclive a los estudios sobre reproducción social, se enfocaba en la explicación que el habitus podía ofrecer respecto a modos de actuar y juzgar fuertemente estructurados en realidades disímiles. Y la cuarta, una revisión -posterior a Meditaciones Pascalianas- sobre sus estudios de Algeria y Bearn, centrados en la corporalidad y en cómo ésta puede servir de insumo y de soporte para la memoria.

En una larga lista de posibilidades, este trabajo comienza distinguiendo los variados usos con los cuales ha sido empleado el término, así como los problemas derivados de la ampliación del mismo en diversos sentidos, a veces incluso contrapuestos. El artículo continúa con una breve presentación de los diferentes usos de habitus en la obra de Bourdieu, seguido de una discusión acerca de cómo el concepto ha sido desplegado en la investigación por sociólogos estadounidenses. La tercera y principal sección se detiene en las aporías que devienen del uso extendido del concepto, y las distintas sendas críticas que han recorrido los especialistas del tema. Esta última sección se centra, no tanto en las críticas que la obra de Bourdieu ha recibido, sino en la tensión que la producción erudita presenta respecto de las explicaciones más disposicionalistas de la acción social. En este sentido presento seis discusiones que apuntan a ciertas conexiones conceptuales o semánticas de habitus que han sido dadas por sentadas, y que han sido retomadas luego por autores como Lahire, Steinmetz, Wacquant, Auyero, y Boltanski (o por autores menos reconocidos que han usado la sociología relacional de Elias para criticar la obra de Bourdieu).

\section{Los diversos significados de habitus en la obra de Bourdieu}

Los múltiples sentidos en que Bourdieu ha utilizado el concepto de habitus vuelven difícil la búsqueda de una fórmula definitiva. Autores recientes (Wacquant, 2004; Gorski, 2013; Decoteau, 2013a; Scheer, 2012 y Steinmetz, 2006) han puesto el acento en su carácter sociosomático, en el encuentro de un cuerpo que conoce y una historia olvidada, reprimida e inconscientemente 
representada (para volver a la referencia marxista al comienzo de este artículo). También podemos pensar en este encuentro como la combinación de una situación particular que enfrenta el agente, las disposiciones particulares que acarrea y los constreñimientos vinculados a las fuerzas colectivas y pasadas que operan en la misma (véase Champagne y Christin, 2012); o usando una fórmula temprana (Bourdieu, 1990), para explicar cómo los agentes resuelven las presiones externas y las contradicciones internas, cómo lo social se externaliza e internaliza, se transforma en un campo de relaciones y cuerpos, y cómo éstos tienen éxito o fallan al intentar ajustarse a los dos momentos o estados.

En un artículo bastante reciente, Crossley (2014, p. 107) vuelve sobre un problema que ya había sido tratado por Brubaker hace tres décadas (1985 y 1993), y que ha motivado desde entonces fuertes críticas: el número de niveles en los que el concepto de habitus opera. Existe de múltiples formas: primarias (como nuestros yo “enclasados”); secundarias (en la socialización profesional); terciarias (por ejemplo, cuando académicos reflexionan sobre sí mismos en tanto encarnación de una práctica particular y el modo en que esta ha sido adquirida). Adquiere forma organizado alrededor de varios principios de división social, algunos más codificados y autónomos, como los campos sociales; otros más difusos y “primordiales” como género, clase o nación. También puede movilizarse inductivamente, como una herramienta de investigación y como un tema, explorando en algunos casos su relación con la historia y, en otros, lo que resulta indispensable para lograr su adquisición y dominio en tanto sentido práctico. Es útil para unificar los múltiples hilos que componen el yo, como también para dar cuenta de las variadas formas en que un agente invierte en sí mismo ${ }^{2}$.Y tiene dimensiones tanto cognitivas como afectivas. La pluralidad de usos, su complejidad interna y su carácter multiescalar -como ya he observado- han obligado a varios comentaristas a “declinar” su empleo (como si un verbo fuese capaz de cambiar según diferentes circunstancias y asuntos) en un sinfín de sentidos. Por lo tanto, si bien es cierto que se torna difícil lograr una definición simplificada y definitiva (como advertía Brubaker, 1993), es fundamental reconstruir cómo el propio Bourdieu ha puesto a jugar el término y a organizar algunas de sus ideas en series ${ }^{3}$.

Como establecía en el párrafo de apertura, podemos ilustrar cuatro períodos y modalidades para habitus en la obra de Bourdieu. La primera aparición sistemática de las ideas de homología y disposición como innovación conceptual (la categoría de habitus estaba ausente aún) se retrotrae al epílogo de una compilación en francés de 1967 sobre la obra del historiador del arte Erwin Panofsky. Allí podemos encontrar ya dos de las dimensiones del concepto que estarán presentes en su variante de largo plazo: la primera es el punto de encuentro, al interior de las prácticas individuales, entre lo colectivo y el contexto epocal; la segunda, el papel de las homologías en la explicación de la trasposición de las disposiciones de un campo a otro. Respecto de la primera, refiere que el habitus es lo que transforma el acervo colectivo -presente en las estructuras inconscientes- en comportamientos específicos en una pluralidad de contextos. Argumenta esta idea demostrando cómo incluso un "genio creativo" puede ser contextuado en su propia época, al comprender cómo sus actos son orientados por el colectivo aunque resulten únicos, singulares en su apariencia ${ }^{4}$. Con el propósito de romper con la idea de “ilusión biográfica”, desarrollará esta línea de investigación en la cual lo individual y lo colectivo se entrecruzan en sus trabajos sobre 
Heidegger y Flaubert, mostrando cómoel modus operandi colectivo se inscribe en la obra de arte como modus operatum. En la oposición entre individualidad y colectivo ya podemos prever una de las principales líneas críticas de la obra de Bourdieu: la tensión entre la reducción de lo individual a lo colectivo o la búsqueda de una nueva alianza entre los dos (Corcuff, 2001).

El concepto aparece en las décadas de 1970 y 1980 bajo dos apariencias, que de hecho pueden ser consideradas como dos caras de una misma moneda, describiendo ambas cómo el habitus produce una disyunción entre las disposiciones individuales y las estructuras objetivas donde agentes despliegan sus prácticas, o cómo ello opera en la confluencia de ambas. Mientras que algunos de los materiales empíricos al respecto ya están presentes en sus primeros trabajos etnográficos sobre Algeria -tal cual reconoce en el prefacio de Algeria 1960 (Bourdieu, 1979)-, como la disyunción entre la dimensión temporal y la estructura económica, es recién con la escritura de Bosquejo de una teoría de la práctica que desarrolla en forma acabada una teoría acerca de cómo ocurre esa disyunción. Como Hage (2013, p. 15) ha señalado, el habitus "es un principio de construcción y adaptación: de esfuerzo por construir un espacio en el mundo donde uno pueda sentirse como en casa”. Por ello, no es de extrañar que en contextos donde ese esfuerzo se vuelve arduo, encontremos los procesos de inercia, disyunción e histéresis que Bourdieu describe en Bosquejo. Y en los contextos donde existe correspondencia entre las disposiciones y el ambiente en las cuales esas prácticas promulgan, podremos observar el efecto de "pez en el agua” que relata en El sentido práctico (Bourdieu, 1990). En ambos escenarios, lo que Bourdieu desarrolla es el carácter dóxico implícito del habitus, como segunda naturaleza, el hecho de que comprender cómo los agentes despliegan estrategias significa entender la lógica difusa, el "sentido del juego", cómo la participación en un campo implica un dominio práctico de los mundos que nos esforzamos por habitar, así como la incongruencia potencial de nuevas condiciones de actualización, como "un pasado que sobrevive en el presente” (Bourdieu, 1977, p. 82). Durante este período aparece también un componente clave para los futuros desarrollos del concepto de habitus: el rol de la hexis, o el carácter encarnado y somático que adquiere, separándolo analíticamente del ethos o su dimensión moral.

En la siguiente serie de usos de habitus encontraremos que las acciones se manifiestan coherentes con el mundo que los actores habitan. Es durante este período que la fórmula de "estructuras estructuradas estructurantes” aparece explícitamente, y donde podemos observar su énfasis en la coherencia de la acción, en tanto conjunto de disposiciones que funcionan plenamente como una fuerza generadora que hace que las acciones se perciban orquestadas o reguladas, al mismo tiempo que improvisadas y sin un conductor/artífice aparente en el horizonte. Este uso aparece claramente en sus escritos sobre las estructuras culturales y trayectorias de distinción del yo (Bourdieu, 1984), en donde se subrayan las conexiones entre las estructuras subjetivas, las posiciones ocupadas en espacios sociales múltiples y las disposiciones al acto. El mecanismo por el cual opera es la transferencia analógica de esquemas de un dominio a otro. Es en Las Reglas del Arte donde su idea de las posiciones y disposiciones que interactúan de manera homóloga en múltiples contextos aparece como campo de posibilidad de ámbitos de libertad, ya que las disposiciones y posiciones no producen naturalmente una sola estrategia, sino más bien un cúmulo de tomas de posición posibles. Por otro lado, es en obras como Las Reglas del Arte, El Sentido Práctico y La Razón Práctica donde Bourdieu incorpora la idea de illusio como un concepto clave para entender la conexión entre 
habitus y campo, llamando nuestra atención sobre cómo el ajuste entre los dos se basa en el sentimiento de implicación de los agentes, impulsados o llamados a participar libidinalmente en un juego ${ }^{5}$.

Es precisamente el concepto de illusio el que me permite pasar a la última serie de usos del concepto, que pone de relieve la conexión entre la creencia y el cuerpo y lo que Bourdieu ha dado en llamar "la crítica de la razón teórica”. Si en trabajos anteriores sobre la actividad intelectual la dimensión clave de habitus destacada era la doxa, y en escritos sobre la producción de clasificaciones profesionales era el ethos, es luego de Meditaciones Pascalianas (y luego de la obra de Loic Wacquant) que hexis se convierte en una dimensión central para comprender la disposición a actuar $^{6}$. Hexis es un término más específico para referir al cuerpo físico socialmente condicionado, sus posturas y gestos y para la investigación de las técnicas a través de las cuales el cuerpo se constituye y es constituido. Bourdieu se sirvió del mismo para revisitar su trabajo de campo en Algeria y Bearn, con el objetivo de explicar los mecanismos por los cuales las experiencias sedimentadas que se encarnan moldean nuestras formas de pensar y actuar, así como nuestros enfrentamientos con las estructuras objetivas. En Dominación Masculina traslada su análisis lejos de la vivienda Kabyle descrita en Bosquejos y muestra cómo la herencia sociosomática está presente no sólo en los objetos, sino también en los cuerpos, como aquello que al inscribirse en el cuerpo de la mujer actúa naturalizando la diferencia entre los sexos, produciendo desigualdad y, en la reproducción a través del cuerpo de esas diferencias, la complicidad de las mujeres en su propia sumisión. En El Baile de Los Solteros Bourdieu ilustra cómo los solteros del ámbito rural encarnan las tensiones entre los cuerpos toscos, torpes de los campesinos solteros locales (son "hombres del bosque”) y las demandas que imaginan de las mujeres con las que solían casarse, que han mudado a otras costumbres y estilos corporales a raíz de su empleo en la economía de servicios de ciudades aledañas a Bearn. Si en el primer caso el cuerpo reproducía la sujeción femenina, aquí imposibilita la reproducción "literal” de esos hombres. El orden social se reproduce inscribiéndose en los cuerpos como un soporte para la memoria (Bourdieu, 2000, p. 161), produciendo diferentes resultados de acuerdo a cómo habían sido o no actualizadas las condiciones bajo las cuales esas disposiciones corporales fueron adquiridas y naturalizadas.

Hace ya más de treinta años Rogers Brubaker (1985) había llamado nuestra atención sobre el uso desmesurado del concepto de habitus. En las dos décadas posteriores a ese llamado podemos ver cómo Bourdieu vuelve sobre el término, lo modifica y elige emplearlo siguiendo el camino original del cual había partido, extendiéndolo en nuevas direcciones. En un escrito posterior (1993), Brubaker invitó a los lectores a pensar el concepto en términos de “disposiciones teóricas” o como un elemento conceptual de una metateoría. Parte del trabajo de difusión y traducción (en Actes de la Recherche, en sus nuevos Prefacios para las ediciones inglesas de sus libros y en los tratados no empíricos donde codificó más adelante algunos de los conceptos vertidos en los trabajos empíricos) señala que el concepto evolucionó no solo para resolver la conceptualización de nuevos problemas empíricos, sino también para proteger al mismo de críticas previas, así como para proporcionar a los lectores instrucciones de su uso, especialmente para aquellos autores que querían adscribir a estas reformulaciones ${ }^{\underline{7}}$. Hasta cierto punto, y como señala Lahire (2001), la proliferación del concepto se encaminó hacia un derrotero de difícil resolución cuando se pretendió controlarla a través de la 
atribución de autoridad a una figura continua, el mismo Bourdieu ${ }^{8}$. Siendo Bourdieu un crítico de la ilusión biográfica resulta paradójico que fuera ese el mecanismo retórico utilizado para establecer jurisdicción y mantener cierto control sobre un concepto que había extendido su alcance hasta un punto en que los límites de su campo semántico se volvían inciertos para los críticos.

Independientemente de estas críticas, los sociólogos se han apropiado del concepto y han dado lugar a una gran cantidad de líneas de indagación. En la sección que sigue, muestro cómo los investigadores radicados en Estados Unidos se han apropiado de esta nutrida herencia.

\section{¿Cómo han empleado los sociólogos estadounidenses el concepto de habitus?}

En un artículo ampliamente citado Charles Camic (1986) discute cómo el carácter pre reflexivo, habitual de la acción social, que ha sido nodal en la concepción de sociología defendida por Weber y Durkheim, ha desaparecido de la sociología estadounidense -gracias a Parsons y los sociólogos de la primera Escuela de Chicago- a medida que la disciplina había magnificado la dimensión intelectual y reflexiva de las prácticas individuales. Poco imaginaba entonces Camic que la sociología disposicional estaba a punto de retornar a los Estados Unidos. Como ha demostrado Gorski (2013), la temprana recepción de Bourdieu en los Estados Unidos se basó en dos textos empíricos que trataban sobre la reproducción habitual, rutinaria e inconsciente de la desigualdad en las sociedades contemporáneas (La Reproducción y La Distinción), y en otros dos textos teóricos (Bosquejos y El Sentido Práctico), cuya lectura tendió a afirmar del mismo modo la primacía de la reproducción sobre la transformación (sobre esto véase también en Calhoun, 2013).

Lamont (2012) y Lizardo (2012) dan una versión ligeramente diferente de la recepción de Bourdieu en los Estados Unidos, aunque también destacan la inclinación “reproductivista” de la recepción temprana. Esta línea fue promovida en Inglaterra, donde los textos de Bourdieu y Passeron abrieron las puertas a un nuevo colectivo de investigadores de la sociología de la educación que siguió sus lecturas de cerca. En cierta medida, la división en los estudios de recepción de Bourdieu es sintomática, ya que denota una relación diferencial de sus trabajos sobre sociología cultural organizacional y cognitiva versus la ramificación académica que es o bien histórico comparativa o etnográfica (los discípulos de Wacquant, Eyal, Steinmetz, Emirbayer y Calhoun), y que se abocó a producir datos empleando categorías bourdianas (autores como Medvetz, Sallaz, Purse, Hanser, Decoteau, Go, Krause, Buchholz, Panofsky, McQuarrie, Desmond y Khan, entre otros). Es como si los trabajos estadounidenses inspirados en Bourdieu se hubieran reproducido en forma separada y paralela por grupos que no establecieron contacto alguno entre sí.

Existe un vasto corpus que utiliza a Bourdieu y al concepto de habitus para promover posicionamientos teóricos sobre la cognición, la acción y la práctica, siguiendo el trabajo de traducción entre el habitus de Bourdieu y los esquemas de DiMaggio (1977). Dentro de esta escuela Vaisey (2009) ha construido un modelo de cognición dual, utilizando habitus para explicar cómo los actores son conducidos principalmente por procesos esquemáticos profundamente internalizados de la conciencia práctica (lo que él llama "el elefante” en la metáfora del elefante-conductor/domador de lo que constituye las tomas de decisión humanas). Luego, en coautoría (Longest, Hitlin y Vaisey, 2013) se abocó a la reconceptualización de los valores como disposiciones transferibles que orientan a los actores hacia la realización de un repertorio de posibilidades disponibles en distintas 
situaciones sociales. Ignatow (2009) emplea habitus para repensar algunas presuposiciones psicológicas clave en la sociología de la moral, con el objetivo de mostrar la naturaleza encarnada de la cognición y el carácter prediscursivo de las competencias socioculturales. Dentro de este mismo encuadre, Lizardo (2009) exploró los orígenes cognitivos del habitus, exponiendo cómo uno de sus efectos clave, la histéresis, puede encontrarse en la obra de Piaget; John Levi Martin (2011) incorpora el concepto en su discusión sobre cómo considerar las explicaciones propias de los actores, con el propósito de mostrar el papel de lo habitual, como una capacidad de “manejar” el mundo, y de proporcionar a los cientistas sociales un elemento clave en la búsqueda de una explicación relacional y estética de las acciones del agente.

Las páginas que siguen se proponen analizar cómo el concepto ha sido utilizado en la investigación actual, en vez de concentrarse en su discusión en el acervo orientado teóricamente. Aunque, por supuesto, se hace bastante énfasis en la reproducción, curiosamente para la sociología estadounidense el concepto ha sido escindido de su íntima relación con las ideas de capital y campo (escisión que Bourdieu desaconsejaba, Bourdieu y Wacquant, 1992, p. 96) y ha sido utilizado para explicar los modos específicos en que contar con una disposición determinada dispone o previene a una persona de actuar de una determinada manera, así como para dar cuenta de cómo es una práctica generativa, constitutiva de la complicidad ontológica entre el agente, sus prácticas y el entorno en que esas prácticas son desplegadas. Para ser sintéticos podríamos decir que la mayor parte de la investigación, o bien se ha centrado en el habitus primario y particularmente en sus efectos en las prácticas de los agentes, o en la adquisición de un habitus secundario. Pocas son las excepciones (Wacquant, 2004 y Desmond, 2007) que consideran la relación entre el habitus primario y secundario y el proceso de transposición de uno a otro.

Entre aquellos autores que utilizan habitus para exponer el carácter generativo de la internalización, el concepto ha sido empleado para explorar el perdurable efecto de raza (tanto en Estados Unidos, como en Brasil o Sudáfrica, entre otros casos posibles), de nacionalismo (en Europa del Este), de clase (en los Estados Unidos) y su intersección, incluyendo asimismo el estudio de la sexualidad (también en los Estados Unidos). En los párrafos que siguen presento algunas líneas o escuelas paradigmáticas en sus propios términos, en lugar de proveer una lista exhaustiva de habitus relativa a cada escuela $\underline{9}$.

Por ejemplo, Phillipe Bourgois y Jeff Schonberg (2007 y 2009) han demostrado cómo las disparidades relativas a la inyección de heroína entre sus métodos subcutáneo e intramuscular pueden ser mejor comprendidas si se conceptualizan como generadas por un "habitus etnizado". En ese sentido, reconstruyen otras prácticas que pueden ser mejor entendidas como parte de la dialéctica entre una disposición relativamente coherente y el modo en que afecta la objetivación: estrategias de generación de ingresos, de parentesco y de presentación de sí mismo. Los autores incorporan la idea de la diferencia disposicional en las dinámicas de poder preexistentes y la memoria de la opresión racial, mostrando cómo incluso entre las personas que han compartido experiencias similares de pauperización por un largo tiempo, los efectos intergeneracionales de la opresión racial aparecen encarnados, produciendo diferencias que pueden luego ser naturalizadas y movilizadas como una fuente de legitimación de la desigualdad.

Alford Young (1999) amplió en su momento las posibilidades de uso del concepto, explorando 
cómo a través de la construcción de fuertes redes de apoyo emocional basadas en la territorialidad, los hombres afroamericanos fueron capaces de acumular capital social en su propio mundo y de navegarlo con relativa facilidad, aunque al hacerlo se vieron impedidos de la posibilidad de acceder, o incluso imaginar, caminos hacia la movilidad ascendente. Annette Lareau $(2002,2003)$ exploró la dinámica entre las prácticas que nos apartan e impiden actuar y desenvolvernos en un ámbito particular donde las diferencias raciales (y de clase) son las más destacadas: las prácticas educativas. Mientras que Young y Bourgois estudiaron la instancia $\mathrm{n}+1$ de las disposiciones, Lareau ilustra el proceso mediante el cual esas disposiciones son puestas en marcha, a través de una descripción precisa y refinada de los estilos de participación de padres en las actividades escolares y de ocio y esparcimiento, mostrando cómo los padres de clase media planifican gran parte de las actividades de sus hijos tanto en la escuela como fuera de ella, fomentan modos particulares de uso del lenguaje, entrenan a sus hijos para intervenir en nombre propio y abogan por un tipo de razonamiento, todo lo cual confluye en propiciar en los niños un sentido de sujeto de derechos y privilegio en lugar de un sentido de restricción frente a familiares, profesionales e instituciones $\frac{10}{}$. A diferencia de Bourgois, ella observa que la clase produce diferencias en un modo que la condición racial no puede explicar por sí misma.

Dos recientes etnografías de Sudáfrica (Sallaz, 2010; Decoteau, 2013a y 2013b) han problematizado e indagado el uso de habitus como una formación racial "pasada en el presente” para dar sentido a la situación poscolonial, donde blancos y negros continúan ejerciendo prácticas en extremo diferentes, a pesar del fin del apartheid legal. Sallaz (2010) ha demostrado cómo, pese a que el mundo que rodeaba a sus informantes -los comerciantes blancos- había cambiado, el dispositivo binario blanco/negro continuó funcionando como el criterio estándar para evaluar y analizar una serie de prácticas comerciales. Decoteau investigó cómo y por qué los sudafricanos negros conviviendo con HIV continúan suscribiendo a una ideología de la salud híbrida, a pesar de los esfuerzos de las agencias estatales por alcanzar un sistema de salud integrado. Al mostrar la disyunción entre la producción y el consumo de salud, se propuso develar la bifurcación e hibridación simultánea del campo de la salud, dependiendo del punto de vista desde el cual se lo observe, si desde la producción o del consumo. En sus escritos, ambos autores invocan el habitus escindido de Bourdieu (Bourdieu et al, 1999; Bourdieu, 2008a): Sallaz lo hace para explicar el fatalismo y la desesperación que experimentan los especialistas en marketing blancos frente al dislocamiento de las relaciones de poder; y Decoteau para dar cuenta del efecto de histéresis (Bourdieu, 1977) resultante del comportamiento de agentes que actúan "como si”, cuando aún se orientan por estrategias mejor adaptadas a situaciones anteriores.

Brubaker y sus colaboradores, en una serie de artículos y en un libro, exponen cómo la etnicidad y el nacionalismo pueden ser pensados en términos de prácticas y conocimientos, o en palabras del propio Bourdieu, cómo la nación se ha vuelto legítima “en tanto principio de visión y de división”. En un artículo del año 2004, Brubaker, Loveman y Stamatov (2004) emplean el concepto de habitus para aludir a los esquemas de trabajo automáticos y rutinarios en reproducir la nación y otras categorías de la práctica “dadas por sentado”, focalizándose en los modos en que el habitus engendra un sentido práctico que regula la acción práctica. En un artículo previo (Brubaker y Cooper, 2000), habitus aparece como aquello que nos permite explicar la autocomprensión de los actores, apartándose de las versiones más cosificadas del concepto de identidad. 
El concepto también ha sido retomado por investigadores en sexualidad. En una serie de artículos, Adam Green (2007 y 2008) desarrolló una aproximación al campo del sexo a través de la conceptualización de los campos sexuales como aquellos que están comprendidos por tres características:

las estructuras del deseo que combinan el habitus erótico de los actores sexuales y sus propensiones sexualmente objetivantes [esto es, aquellos aspectos de las «disposiciones duraderas» que adjudican un valor erótico a un objeto dado, y que son ellos mismos producidos por sistemas de estratificación], la historia cristalizada de las relaciones de estos actores en términos de niveles de atracción sexual y la distribución del capital erótico entre los actores de un lugar físico determinado de sociabilidad sexual (Green, 2008, p. 30).

Esto le permitió subrayar el carácter estructurado, encarnado y estratificado del deseo, revelando el esquema racial derivado de los sistemas históricos de clasificación y poder a través de los cuales varones homosexuales blancos de clase media les asignan un estatus erótico a los varones negros hipermasculinizados $\underline{11}$.

Otras dos investigaciones apuntan también a explorar el habitus principal o maestro, pero, para ello, lo colocan de revés, tratándolo como el ensamblaje de una performance de disposiciones particulares, en lugar de predisponer o inhibir a los agentes a actuar de un modo particular. Hancock

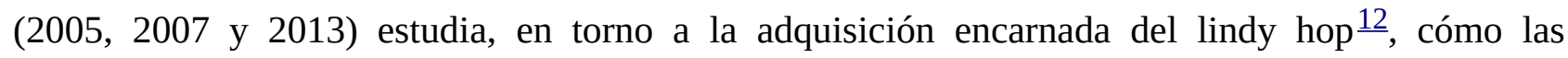
mitologías raciales enquistadas y naturalizadas en nuestro esquema mental y conceptual del mundo son reproducidas, actualizadas y materializadas por medio de la práctica cultural de la danza a través de nuestros cuerpos. A partir de ello, propone un modelo alternativo para explicar la identidad racial, basada en las competencias y tipos de conocimiento incorporados que se despliegan en la práctica. Caputo (2012) se introduce en el mundo de una agencia de empleo que provee servicios a expresidiarios para comprender cómo los comportamientos aprendidos para desenvolverse en prisión (lo que ella entiende como un habitus carcelario) resultaron inadecuados para el nuevo contexto de reinserción social. Mientras que el objetivo del trabajo es explorar cómo la reinserción se ve dificultada por las respuestas incorporadas a la violencia interpersonal una vez afuera, el artículo complejiza a su vez las visiones sobre lo que constituye la disposición primaria de una persona luego de un largo y profundo proceso de reeducación corporal $\underline{13}$.

Este último artículo hace de eslabón para comenzar a describir la segunda y fructífera variante a partir de la cual el concepto de habitus ha sido empleado: las técnicas rutinarias del cuerpo que construyen competencias y fomentan la complicidad ontológica con el mundo. Luego de la publicación de Wacquant (2004), se produjo una explosión de investigaciones hacia finales de la década del 2000, de artículos y libros focalizados en disposiciones secundarias y especializadas como las de políticos, sopladores de vidrio, luchadores aficionados, bailarinas de ballet, pianistas, especialistas en artes marciales $\frac{14}{4}$; así como de las primarias: investigaciones sobre las rutinas de oración musulmanas, estudiantes de escuelas secundarias o de "pacientes del estado".

Entre aquellos que profundizaron, no en la idea del habitus como creador de prácticas, sino en cómo las prácticas crean la estructura de una disposicionalidad, se destaca especialmente el trabajo de O’Connor (2005, 2006 y 2007) ya que amplía las interacciones entre el yo y el otro, tan centrales a 
las técnicas del cuerpo, hacia el papel de los objetos como instrumentos. En su trabajo sobre los sopladores de vidrio ella ilustra en detalle la experiencia fenomenológica de adquirir y transmitir el conocimiento práctico y tácito. Al reponer cómo el calor juega un papel importante, nos informa de las limitaciones impuestas por el mundo material sobre el trabajo de las disposiciones y la dialéctica entre ambas. Mahler (2008) nos acerca aun tipo particular de mundo, al conocimiento práctico de los políticos de carrera y de las convenciones ocultas y encarnadas de la competencia y el conocimiento que hacen posible afrontar con destreza la actividad política diaria. Dicho trabajo, que relata cual novela de aprendizaje cómo un miembro del equipo de campaña aprende sobre política durante el día anterior a las elecciones, se opone a la idea, a la que solemos tender, sobre el conocimiento del político como puramente intelectual, incorporando la dimensión encarnada de esos saberes. Su investigación contribuye a resaltar lo que él llama "el círculo hermenéutico estrechamente unido entre el agente y el mundo" 15 .

En un conjunto de investigaciones, Bryan Turner y sus colaboradores (Turner y Wainwright, 2003) han estudiado los efectos de las disposiciones en la consolidación del espíritu de cuerpo de los bailarines individuales, y de sus consecuencias respecto a la consideración que hacen de sus lesiones corporales como algo que es parte de los efectos "naturales”, inherentes a su vocación. En un segundo artículo muestran cómo el pertenecer a compañías específicas diversifica aún más los estilos de corporalización, forzando a los bailarines profesionales a reaprender sus habilidades corporales de un modo reflexivo cuando mudan de una compañía a otra (Wainwright, Williams y Turner, 2006) ${ }^{16}$. En un estudio anterior, Bob Alford y Andras Szanto (1996) confrontan los mundos profesional, pedagógico y médico para develar la naturalización del dolor en la adquisición de los saberes y habilidades pianísticos. Al restituir el proceso emergente de disputa y la formación de nuevas técnicas para el reaprendizaje sin dolor, el artículo señala las tensiones entre las condiciones de adquisición y su despliegue bajo condiciones distintas en las cuales resultan desnaturalizadas.

Para comprender la reconstitución del habitus primario, Dan Winchester (2008) explora cómo un grupo de adultos produjo nuevas subjetividades morales a partir de la movilización de prácticas religiosas incorporadas. Los musulmanes conversos que investiga se involucran en competencias como el ayuno y la oración para desarrollar virtudes morales. De esta manera, Winchester muestra cómo sus fieles se restructuran en torno a la práctica de disposiciones hacia el espacio y el tiempo incorporadas en el actor social, transformando cualitativamente sus subjetividades. Las prácticas que distingue (salat, ayuno) sirven para ilustrar cómo estos conversos se involucran en prácticas que reorganizan su memoria corporal y sus patrones temporales, cómo esto se relaciona con el desarrollo de un sentido de la humildad, de la modestia y de la deferencia hacia la autoridad, ya que necesitan de esto para responder al tiempo de Dios cotidianamente, ycómoencuentran, en la disciplina recién descubierta, la producción consciente de un yo que puede servir a Dios.

A diferencia de este caso, en el cual habitus es usado para iluminar un proceso de autopoiesis, el trabajo de Auyero (2012) sirve de contrapunto: los métodos insidiosos bajo los cuales las instituciones estatales en la Buenos Aires contemporánea ponen en práctica lo que él llama "el modelo de paciente”, una disposición que obliga a los pobres a esperar. Su libro esboza los procesos a través de los cuales la temporalidad participa en la (re)producción de la dominación. Auyero se pregunta, ¿qué tipo de proceso es la sumisión a la espera? Para responderlo nos ofrece un catálogo y 
una cronografía $\frac{17}{}$ de la dominación (retomando otro de los esfuerzos de la obra de Bourdieu) que revela cómo las personas pobres naturalizan la idea de que para obtener algo de los agentes estatales deben esperar, aunque a veces no sepan ni cómo ni por qué lo están haciendo.

El trabajo de Kahn (2001) constituye una tercera etnografía, intermedia en la distinción que podemos imaginar organizando las dos anteriores entre la adquisición consciente/inconsciente de ciertas disposiciones. En su estudio sobre cómo la escuela secundaria forma comportamientos de élite, usa habitus (o como él lo llama "la adquisición de la habilidad corporal”) para explicar la facilidad con la que el privilegio se adquiere y actualiza en una serie de rutinas corporizadas. Su contribución más importante para la comprensión del habitus es su reconstrucción sobre cómo la encarnación de un yo relativo al género y de otro vinculado a su condición de privilegio impone presiones contradictorias sobre los cuerpos de mujeres de élite en la escuela $\underline{18}$.

Si bien todos estos trabajos han explorado los modos en que un habitus primario predispone a los agentes a actuar o los aleja de estrategias particulares, el trabajo pedagógico necesario para adquirir el dominio práctico de un mundo secundario, o la reestructuración del yo producto de la adquisición de una disposición secundaria, pocos investigadores han realmente articulado plenamente la relación entre un habitus primario y secundario, analizando la transposición, la refracción y las transformaciones que se producen en ese movimiento. El estudio más conocido que combina estas dos líneas de investigación es la etnografía de Loic Wacquant del habitus pugilista. En su libro Entre las cuerdas (y en múltiples artículos) logra combinar una microsociología carnal de los procesos de iniciación, aprendizaje y pertenencia a través de los cuales jóvenes afroamericanos provenientes de barrios suburbanos aprenden el oficio del boxeo con un análisis macrosociológico e histórico sobre cómo el gueto actúa como un "instrumento de cierre racial y de dominación social” (Wacquant, 2011, p. 85). Utilizó la oposición entre la calle y el ring de boxeo para representar metafóricamente el sentido de contigüidad, continuidad y ruptura entre las disposiciones que los jóvenes traen del gueto y la disciplina a través de la cual son readaptadas, empleando esas cualidades en la búsqueda de una trayectoria diferente (Wacquant, 2004, p. 55). A través de esta oposición, muestra cómo el gimnasio traduce la cultura masculina de la resistencia física, el honor y la violencia corporal por medio de un entorno regulado, entrenándolos de forma tal que los intercambios agonísticos del afuera son reconvertidos en una violencia simbolizada y controlada, aunque aun físicamente palpable. Su trabajo, como han remarcado él mismo (Wacquant 2011, 2014a y 2014b) y otros (Body and Society 2013 y 2014) ha contribuido a extender, refinar y concretizar la idea de habitus, tanto como un tema de investigación -el estudio de sus propiedades generativas y de su adquisición- como de una herramienta de investigación -ya que la inmersión carnal que sostiene es una vía clave para el estudio de la adquisición de un dominio práctico secundario-.

Mientras Wacquant muestra cómo la continuidad y la contigüidad son reconstruidas por medio de prácticas disciplinarias en algo casi completamente diferente, el trabajo de Matt Desmond (2006 y 2007) subraya el ajuste extraordinario entre los dos mundos habitados por los agentes que estudia, los bomberos forestales. Su investigación postula que el habitus general de lo que él entiende como “el muchacho de campo" se convierte en el habitus específico del bombero, centrándose principalmente en cómo los agentes perciben y controlan el riesgo. Ampliando el argumento de 
Meditaciones Pascalianas, el autor hace de su trabajo una reflexión acerca de qué es lo que los agentes traen consigo, como individuos estructurados en torno a su habitus general, qué es lo que permite que esas subjetividades puedan experimentar ligeras modificaciones y ajustarse a un habitus estructurado específico sin mayores inconvenientes. Si Wacquant plantea una división entre la calle y el gimnasio, Desmond muestra cómo sus informantes estructuran el mundo a través de las oposiciones binarias adentro/afuera y campo/ciudad. En contraposición a la ilusión de la autodeterminación (personas que aman el riesgo se orientan hacia las profesiones más cautivantes), el trabajo postula que en tanto "muchachos de campo" -y en su condición de bomberos- ya se encuentran mejor predispuestos a enfrentar los riesgos del afuera, aunque una parte importante del argumento radica también en cómo el servicio forestal logra generar lealtad y confianza, transmitiendo un modo de actuar en el cual el fuego es considerado como algo inofensivo antes que peligroso, reelaborando esas oposiciones binarias originales.

\section{Las Aporías del habitus}

En este apartado de cierre, quiero hacer un balance sobre los variados usos de habitus que he presentado, a partir de la revisión de seis discusiones que se han suscitado en torno al concepto. Parte de lo que está en juego en esas discusiones es cómo investigar cada una de las conexiones que han sido dadas por sentado entre las distintas partes constitutivas que componen el habitus (las conexiones entre el habitus y cómo es adquirido, entre las disposiciones y su génesis, entre trayectorias y disposiciones, entre lo individual y colectivo, entre disposición y contexto); además, dependiendo de la relación de los autores con el concepto, sus intervenciones pueden dar lugar a intentos por refinarlo o ganar mayor especificidad, por extender la conceptualización, ollevar a su abandono total.

El primer debate ha sido sobre el tipo de trabajo metateórico que el concepto de habitus induce en las explicaciones de Bourdieu sobre la acción social y en la relevancia del problema de la estructura y la agencia. Este punto puede resumirse mejor en las múltiples afirmaciones que se han hecho del habitus como una caja negra que actúa retrotrayendo el debate del nivel micro-macro a un estadio anterior, sin claridad alguna sobre cómo y por qué los agentes eligen entre las opciones alternativas establecidas por el habitus (Boudon, 1998; Van den Berg, 1998), como una especie de realidad mística superpuesta inferida antes que observada (DeCerteau, 1984), reificada/cosificada como factor explicativo (Crossley, 2014), utilizado como una deus ex machina (DiMaggio, 1979), o la bête noire de la sociología bourdiana (Boltanski en Dosse, 1997) $\underline{19}$. La crítica que todos estos autores comparten hacia la escuela bourdiana es que da por sentadas ciertas homologías entre disposiciones y posiciones, y entre posiciones de diferentes campos, sin aportar descripción empírica alguna de las matrices y disposiciones, o cualquier especificación de cómo comprobar estas empíricamente $\underline{\underline{20}}$.

Mientras que algunas de las primeras críticas no previeron el movimiento conceptual posterior desde la inculcación y reproducción hacia la corporalización y disposición-, las críticas sobre la falta de especificación de los mecanismos internos del habitus han sido respondidas en su mayor parte por el movimiento posterior a las investigaciones de Wacquant, que mueve al habitus de su estatuto como una construcción teórica a un objeto empírico a ser de y re-construido, y como un 
modo de orientar la investigación cualitativa. Aunque es cierto que varios de los investigadores inspirados en Bourdieu todavía continúan utilizando el término en el sentido de una disposición homogénea que dan por sentada (véase por ejemplo en la literatura sobre el habitus racial), una gran cantidad de investigadores recientes, como he mostrado, han emprendido la labor de otorgar mayor especificidad a las disposiciones de los actores a través de la exploración situada de sus hábitos de pensamiento y acción. Esto se ha logrado en parte gracias al seguimiento del pasaje en los estudios del propio Bourdieu de un primer enfoque sobre la mente y la cognición a uno sobre el cuerpo 21 . Por otro lado, el reciente trabajo epitextual de Wacquant (2011, 2014a y 2014b) parece haber tomado muy en serio la crítica del habitus como caja negra, tal es así que utiliza alguno de los términos de la Teoría de Acción en Redes (ANT) para explicar la articulación del habitus. En este sentido afirma que “abre la 'caja negra' del habitus pugilístico revelando la producción y el ensamblaje de las categorías cognitivas, las habilidades corporales y deseos que juntos definen las competencias y aspiraciones específicas de un boxeador” (2011, p. 87). El triple juego entre, por un lado, el movimiento que Bourdieu realiza enfatizando con el correr del tiempo la corporalidad por sobre las estructuras cognitivas, la proliferación de investigaciones posteriores a Wacquant que exploran el ejercicio de adquisición de un habitus secundario, y la publicación póstuma de los primeros trabajos que muestran a un Bourdieu autoexplorando el "montaje” de su propio habitus, y de aquellos referidos a sus temas de investigación preliminares en Algeria y Bearn, le hado un carácter más concreto y observable al concepto yal trabajo explicativo que se le pide.

Los siguientes debates están centrados, no tanto en si efectivamente tiene sentido pensar en términos de disposiciones, sino en cómo operan esas disposiciones. Para el segundo debate me gustaría introducir la cuestión sobre cómo las disposiciones son transmitidas. Esto puede ser desarrollado a partir de dos discusiones específicas: a) la primacía de la adquisición irreflexiva, y b) las transposiciones de esquemas de un contexto al otro.

En la primera, etnógrafos interesados en el habitus secundario han evidenciado momentos en los cuales la adquisición se vuelve permeable a la conciencia y la discusión explícita, o han deliberado sobre el trabajo reflexivo tanto del cuerpo como del "sentido del juego". Corcuff (2007) ha retomado el trabajo de Faure (2000), quien estudió la formación de bailarines de danza clásica, para ilustrar cómo junto a los automatismos corporales implícitos, existen también momentos de prácticas lingüísticas (diferentes formas en que sus profesores intervienen) y momentos de reflexividad (mirándose a sí mismos frente al espejo, “calcando” paso a paso el próximo ejercicio), enfocándose en cada caso en las variadas lógicas prácticas en juego que apelan a diferentes grados de reflexividad. Downey (2008), en su estudio sobre las comunidades que practican capoeira, señala asimismo al "andamiaje" (scaffolding)como un modo explícito, repetitivo, autoconsciente de adquisición de saberes corporales.

En la segunda discusión, encontramos sociólogos de la cultura y la educación que se han interesado por la naturalización y universalidad de los esquemas de transposición. Algunos se han ocupado de las múltiples aristas a través de las cuales aparecen la analogía y la transposición en la obra de Bourdieu, reconociendo un sinnúmero de prácticas no siempre coherentes en una lógica superpuesta (DeCerteau, 1984). Otros han estudiado la circularidad de los objetos y los sujetos articulados en torno a la idea de homologías, excluyendo la posibilidad de múltiples trayectorias de desviación 
(Benzecry, 2011). Del mismo modo, Bernard Lahire ha analizado la especificidad de aquello que puede ser transferido de los modelos preexistentes de socialización; Lahire (2011, 2006 y 2003) se concentra, por ejemplo, en el estudio caso por caso de qué es lo que se transmite de la socialización educativa al medio profesional: ¿̇son las relaciones interpersonales?, ¿los usos de un saber “universal”?, etc.

Es precisamente en la sociología disposicional de Lahire donde podemos encontrar un interlocutor clave para el tercer debate iniciado luego del habitus bourdiano: ¿cuál es la génesis del habitus? ¿Qué aspectos del pasado resultan privilegiados en la lógica de la repetición y en los modos en que los sujetos actúan en escenarios novedosos? Dicho contexto inicial, ¿̇actúa como una cicatriz o como una marca de nacimiento? Autores tan diversos como el mismo Lahire $\underline{22}$, Green, Steinmetz, Widick y Decoteau han abogado todos por ir más allá de una comprensión cognitiva del habitus y su lógica de habituación, sus desencadenantes, respuestas y entornos $\underline{23}$, observando el lenguaje psicodinámico que Bourdieu adoptó en sus últimos trabajos, con el propósito de traer a discusión nuevamente temas o términos como libido, relación de objeto, inversión, catexia, deseo, fragmentación del yo, represión, denegación, repetición, la tensión entre lo simbólico y lo imaginario, etc. Todos estos autores exploran en profundidad lo que significa pensar en el inconsciente como una dimensión fundamental para proveer explicaciones sobre cómo funcionan los cuerpos, cómo están articulados simbólicamente y cuáles son sus trayectorias y posibilidades. Steinmetz (2006 y2013) y Decoteau (2013a) se detienen en el trabajo de unificación que el habitus realiza sobre el sujeto, y en cómo las cuestiones relativas a la fragmentación -que no suelen reconocerse dada la unidad presupuesta de las disposiciones- pueden ser mejor explicadas si vinculamos los conceptos bourdianos con la tensión lacaniana de un sujeto constituido (y estructurado) dentro de un orden simbólico, o de una modalidad imaginaria, en la cual ámbitos y acontecimientos desarticulados son considerados como unificados $\underline{\underline{24}}$.

De modo similar, con el objeto de construir una sociología del deseo, Green (2007 y 2008) se interroga por la noción de un habitus de género y cómo esta puede ayudarnos a comprender el modo en que los factores sociológicos intervienen en la constitución de fantasías sexuales, explorando los paralelismos entre relaciones de objeto y cómo las relaciones sociales son somatizadas con la intención de alejarse de conceptos sociológicos tan estimados por la teoría de género como libretoy actualización del rol. Green indaga en la internalización de objetos sociales a través del habitus, el cual objetiva inconscientemente la percepción individual de los cuerpos potenciales existentes, incluyendo el suyo, y opera en consecuencia, derivando en la somatización del género. Widick (2003), en cambio, se concentra en buscar correspondencias con Freud, complementando el habitus de Bourdieu con una teoría de la identificación de género, donde el modelo de ideal masculino de los agentes de bolsa (el Súper Agente) es lo que nos ayuda a entender mejor el apego, la "segunda naturaleza” y el “instinto comercial” que desarrollan a medida que aprenden su oficio.

Lahire (2001 y 2011) propone una sociología piscológica orientada a la articulación de las explicaciones disposicionalistas con una argumentación freudiana (vía su divulgador francés, Laplanche) sobre la intravariación individual. Al énfasis de Bourdieu sobre el habitus como una matriz que soporta experiencias pasadas en el cuerpo del individuo, Lahire agrega dos advertencias: en primer lugar, una pregunta por los motivos por los cuales nuestras disposiciones operan en 
algunos contextos, pero fallan al actualizarse en otros; y, en segundo lugar, dadas las variadas experiencias de socialización que coexisten dentro de un mismo cuerpo -y su carácter inconsciente-, ¿cómo sabemos cuáles de ellas son privilegiadas? ¿Cómo sabemos de qué modo intervienen posteriormente en la vida de un individuo (véase Frére, 2004)? En ese sentido, Lahire se opone a un postulado de la presupuesta homogeneidad del habitus: si los individuos responden a múltiples contextos y experiencias a través de un repertorio heterogéneo de disposiciones, entonces, ¿cómo determinamos cuándo opera cada una y por qué?

Esto nos lleva al cuarto debate introducido por el concepto de habitus (en este caso, el trabajo de Bourdieu sobre Heidegger y Flaubert): ¿cómo llegamos a lo singular? ¿cómo podemos iluminar una trayectoria individual enmarcada en un grupo o época y explicarla con las herramientas de una sociología disposicionalista? Algunos de los críticos de Bourdieu (Corcuff, 2001; Lahire, 2001) han abogado por el trabajo de Elias sobre Mozart, al considerar que logra ilustrar la forma en que las primeras relaciones psicosociales orientan luego a los agentes en sus vidas y en sus prácticas de un modo particular. Al análisis de Bourdieu sobre Heidegger, estos críticos oponen la interpretación que Elias realiza sobre la vida de Mozart (Elias, 1993), en la cual subraya la relación de Mozart con su padre, la interacción entre la intimidad y la distancia al interior de la sociedad cortesana debido a su rol como músico, su posterior partida y su trabajo como músico independiente, y el modo en que su estructura subjetiva se hallaba fuertemente anclada a una contradicción entre la fama y la humildad de su hogar. El argumento de Bourdieu se concentra, en cambio, en articular un modelo disposicionalista de matrioskas -las muñecas rusas huecas que albergan otras muñecas de menor tamaño- preguntando primero por la clase social del filósofo alemán, luego por su fracción de clase, más adelante por su oficio de filósofo, y por su lugar en un campo específico y en un campo de ideas en general. $\mathrm{Y}$ todo ello lo hace sin decir mucho acerca de su socialización familiar y académica, sus orientaciones sentimentales, políticas y religiosas, dando por sentado una vez más que las disposiciones analizadas (y no las otras propuestas) son la clave explicativa de la génesis del habitus de Heidegger.

Este programa de investigación no se limita a una crítica de Bourdieu, más bien intenta repensar qué podríamos decir sobre una variación intraindividual. Lo que le objetan a Bourdieu es que, si bien puede explicar la presencia del colectivo en el sujeto, no da cuenta de las desviaciones minuciosas de un individuo respeto de un grupo, o cómo los agentes cambian con el correr del tiempo, ganando espacios de socialización diferentes y contrapuestos; también apuntan contra la supuesta homogeneidad de la socialización familiar. Para responder a esto, Lahire (2011, p. 32) formula la idea de stock, centrando su atención en la incorporación de múltiples esquemas de acción y hábitos, organizados en torno de varios repertorios de actuación y de los contextos adecuados para su despliegue. También hace alusión al fenómeno de la disonancia cultural (2008), como aquel que nos permite observar la tensión entre diferentes modos de socialización y de gusto incorporados. Para distinguir el primero-el concepto de stock- de la idea de campo, repone un conjunto de ejemplos inspirados en su análisis sobre la “doble vida” de los escritores de literatura, mostrando cuán poco de lo que realmente hacen está confinado dentro de los límites de un campo profesional, y cuánto más se explica por los elementos ajenos a este y por los ajustes que deben realizar cuando dejan el "juego" y entran en otros contextos de acción relevantes (familia, enseñanza, trabajos desvinculados al quehacer literario, traducciones, etc. $)^{25}$. 
Una línea de investigación afín ha comenzado a explorar cómo recuperar las definiciones propias de los actores, los sentidos del soi meme, los proyectos del sujeto, apartándose de su descalificación inmediata como ilusiones biográficas. Autores como Dose (1997), Lahire y Corcuff (2007) en Francia, y Neil Gross (2003) en Estados Unidos, han señalado todos que mientras el trabajo de Bourdieu es extraordinario para responder a la pregunta por la continuidad en el tiempo del carácter de una persona (gracias al habitus individual), no ha ofrecido del mismo modo respuestas sobre aquello que constituye la segunda pieza de la identidad de un sujeto: los sentidos de la subjetividad del actor (como cuando éste se pregunta, ¿quién soy?) y cómo ello contribuye a comprender cómo los agentes orientan sus acciones.

Esto último sirve de nexo con el quinto debate abierto gracias a la sociología disposicionalista de Bourdieu: la pregunta sobre qué aspectos de lo local o del contexto inmediato activan o inhiben disposiciones, o hasta qué punto el hecho de poseer una habilidad determina lo que los agentes hacen. Aquí me gustaría ir más allá de la referencia a la obra de Lahire (quién lo ha conceptualizado teóricamente) con tres ejemplos de Auyero (2009), Hirschkind (2001) y mi propio trabajo (Benzecry, 2011). Mientras que para Bourdieu una de la causas de transformación de las disposiciones se halla en la situación de incongruencia entre la condiciones de operación del habitus y las de su adquisición -situación que deviene en un cambio o transformación a medida que se “desnaturalizan”, por lo cual un entorno transformado debería resultar en la eventual transformación del modo en que se haya representado-, lo que estas tres investigaciones enfatizan es cómo esas discrepancias son la fuente de la continuidad, de la reproducción social, en lugar del cambio. Los fanáticos apasionados de la ópera se asientan sobre un pasado perdido para reproducir parcialmente una actitud hacia la ópera surgida de ese mismo pasado, persistente en y a gracias a las acciones y discursos del presente. En lugar de encontrar un ajuste casi automático entre circunstancias, disposición y práctica, lo que hemos aprendido del análisis empírico es que los recursos comunitarios, la sociabilidad al interior del grupo, y el aislamiento respecto de las interpretaciones rivales son todos ellos factores claves en la mediación de la producción del ajuste o discrepancia entre los recursos y la práctica.

Mi trabajo avanza dos críticas adicionales hacia las explicaciones disposicionalistas del consumo cultural: a) que la variación en el apego al mismo producto o práctica cultural puede ser explicado solamente por las trayectorias preexistentes -por el contrario, los fanáticos de la ópera en Buenos Aires se diferencian internamente a partir de categorías creadas a través de las prácticas de apego, las cuales encajan difícilmente en posiciones preexistentes o son de difícil intercambio en otros contextos de sentido-; y b) que la circularidad de las explicaciones homológicas que recuperan las condiciones de producción de un objeto cultural son necesarias y suficientes para dar cuenta en forma acabada de su consumo. Si bien esas condiciones de producción generan una efectividad condicional, no resulta claro cómo esta se actualiza luego (o no), cómo el horizonte de sentido inscrito en la obra es movilizado $\underline{\underline{26}}$.

En la misma línea, Auyero y Swistun (2009) estudiaron la relación entre habitus y hábitat, adentrándose en el mundo tóxico de Villa Inflamable y observando el modo en que sus residentes interpretan su contaminado entorno. Los autores ilustran convincentemente cómo las percepciones de aquellos involucrados en ese mundo son articuladas por un “trabajo de confusión” concertado 
por actores externos. El trabajo de antropólogos como Hirschkind (2001) muestran cómo, en lugar de la transformación automática y el abandono de la relación entre la disciplina ética y el discurso islámico, debido a las demandas de empleo y la dispersión geográfica de sus practicantes, lo que vemos en la gestación de un público insurgente, contracultural en Egipto revela cómo los propios actores, gracias a la tecnología de los sermones en cassettes y la sociabilidad grupal que produce, se vuelven activamente ciegos a las transformaciones de las condiciones bajo las cuales esas disposiciones éticas debían operar. Esto añade una mediación más a la relación entre esquemas y recursos, y vuelve nuestra atención sobre el papel que juegan los grupos en el mantenimiento del poder productivo y reproductivo de las disposiciones.

Si bien el sexto debate es tributario del anterior, nos permite traer a un primer plano a otra de las Escuelas Francesas que han arribado luego de Bourdieu $\underline{27}$ : la sociología pragmática. Lo que estos autores quieren saber es, si explicar la acción social se trata efectivamente de descubrir el encuentro entre las múltiples potencialidades del cuerpo y las múltiples potencialidades del entorno inmediato, ¿qué hacemos con la pluralidad de las disposiciones?, ¿qué hacemos con las múltiples esferas de actividad en las cuales se despliegan y afinan nuestras prácticas? El trabajo de Boltanski y Thévenot (1999; 2006) y Boltanski (2012) aborda estas cuestiones postulando la pluralidad de modos de compromiso y ajuste de la acción, e indagando en ellos en términos de "repertorios" de recursos culturales que son movilizados en diferentes escenarios. Con el objetivo de ir más allá de la teoría de Bourdieu sobre la reproducción social anclada en la interacción entre disposiciones y campos, los autores sustituyen ambos términos. En lugar de las disposiciones, introducen la idea de competencias críticas del actor -integradas por elementos a la vez mentales y corpóreos-, las cuales son desplegadas y "testeadas" por su competencia en conflictos cotidianos; las habilidades y capacidades no son potencialmente determinantes, más bien son elementos que pueden ser utilizados o no, dependiendo de cada situación.

Lo que distingue una competencia de una disposición es el hecho de que los actores movilizan diferentes formas de justificación a lo largo del tiempo, y que en una situación dada los actores pueden contar con una pluralidad de justificaciones a mano. En lugar de un campo, define la existencia de múltiples órdenes macroestructurales de valor, aunque éstos no predefinan ni determinen los conflictos. Dado que los conflictos están abiertos a diferentes formas de justificación, y que a través de ellos el autor reconoce las tensiones entre diferentes órdenes de valor, son los actores los que basan sus justificaciones en esos órdenes, tanto para confirmar como para rechazar declaraciones contrapuestas. Al igual que en Lahire, encontramos aquí otra versión del argumento sobre cómo y cuándo las disposiciones son activadas o inhibidas, y también otra versión de la idea del agente a la vez multisocializado y multideterminado, solo que en este caso el peso recae en cómo determinadas situaciones pueden corresponderse mejor con ciertas disposiciones en lugar de otras, anulando la idea de una lógica disposicional unitaria, transferible a través de cualquier contexto de acción.

\section{Conclusión}

Como bien ha señalado Frére (2004, p. 95), gracias al trabajo de la sociología psicológica y de la 
sociología pragmatista francesa sabemos más acerca del funcionamiento subjetivo del habitus. Esto debería ayudarnos realmente a refinar y revigorizar las explicaciones disposicionalistas, ya que en efecto la apertura de las cajas negras nos permite ir más allá de los enfoques en los que el habitus se ha dado por sentado, o -por esa razón- desechado.

El artículo tiene también una ambición más modesta: que sea posible -gracias a la exploración de sus diferentes usos y críticas- especificar aún más cómo y cuándo las personas usan habitus, y profundizar la discusión sobre el tipo de preguntas de investigación (lo que Abend [2008] describe como una terapia semántica). El concepto se ha extendido -gracias tanto al trabajo de las personas que se encuentran dentro de la galaxia Bourdieu, como de aquellos que se lo han apropiado parcialmente- hasta el punto en que ahora se ha convertido en algo a ser invocado sin especificación alguna, junto a otros conceptos mayores de la sociología con una historia conflictiva, como estructura, cultura o agencia. La consecuencia de haber alcanzado este estado amorfo es que las explicaciones disposicionalistas se han vuelto tan centrales que pocos investigadores están ofreciendo alternativas explícitas al realizar indagaciones empíricas. El hecho de que haya una falta de debate entre las diferentes posiciones en pugna que utilizan el concepto (investigadores interesados en la cognición, formaciones históricas comparativas, la reproducción somática del yo, la intersección entre la multiplicidad del yo y su unidad de acción) debería llamar nuestra atención sobre cuánto de habitus se ha convertido en un cuasiobjeto (Latour, 1993) que enrola personas -en un aparente diálogo- que de otra manera bien podrían estar hablando de cosas muy diferentes. Si bien en ello radica su fortaleza "funcional”, debemos preocuparnos por saber si estos investigadores están hablando en absoluto de la misma cosa. Parece ser que, a esta altura, ir más allá del concepto de habitus no es solo una cuestión de crítica y reconstrucción -como en la mayoría de los programas sofisticados que presenté-, sino de especificar a qué nos estamos refiriendo cuando hablamos de disposiciones.

\section{Notas}

1 Título original: "Habitus and beyond. Standing on the shoulder of a giant looking at the seams”. Artículo traducido por Santiago García Martín (CISH-IdIHCS-CONICET-UNLP).El traductor agradece a Enrique Garguin y Julia Hang por sus aportes a la tarea de traducción.

$\underline{2}$ Para una lista completa, bien explicada y organizada de habitus, véase Wacquant, 2011, 2014a y 2014b.

$\underline{3}$ Dado que existen entradas específicas para habitus en este volumen, lo que aquí presento no posee el nivel de detalle que amerita; esta sección está aquí para volver más adelante sobre algunos de los límites y aporías que los críticos han señalado.

4 Estará presente en sus futuros trabajos sobre la élite francesa y su reproducción a través de los campos cultural y educativo (Bourdieu 1974, 1984 y 1998), donde desarrolla la tensión entre la socialización primaria, el papel de la escolarización y del esprit de corpsprofesional que aparece en nuceen sus comentarios sobre Panofsky.

$\underline{5}$ Sólo aquellos que han incorporado el habitus específico de un campo son capaces de jugar correctamente. 
$\underline{6}$ En sus intercambios con Wacquant (Bourdieu y Wacquant, 1992, pp. 172-3, referentes a su trabajo sobre la dominación masculina publicado en Actes de la Recherche) anticipó la relación entre cuerpo y socialización, pero no la desarrolló plenamente.

Z Deberíamos preguntarnos si la escasez de monografías de corte bourdianas en Estados Unidos -a pesar de la amplia citación de sus conceptos- no se debe a la tensión entre cuan extendido está el uso del concepto y a las condiciones estrictas bajo las cuales se considera apropiado su uso.

8 Como explica Foucault (1969), la función-autor “pone un límite a la proliferación peligrosa de significados en un mundo donde se es mesurado y prudente no sólo con los recursos y riquezas propios, sino también con los discursos y sus significados. El autor es el principio de ahorro en la proliferación del significado”.

9 Wacquant (1993) sigue siendo una gran referencia para reunir un inventario sobre las limitaciones o deficiencias de la traducción estadounidense de la primera recepción "reproductivista” de Bourdieu. He procurado trabajar en esta sección con la producción posterior a su intento de revisión.

10 Otras investigaciones sobre educación, clase y status como la de Stevens (2007) reconocen el carácter bourdiano implícito en su etnografía, aunque no utilizan directamente su marco teórico.

11 Discutomás adelante su artículo sobre el habitus erótico como teoría de relación de objetos.

12 Nota del traductor: El lindy hop es un estilo de baile estadounidense que se desarrolló originalmente en Harlem, Nueva York, en los años 1920 y 1930 y que evolucionó a la par de la música jazz de la época.

13 Aunque se inspira en la obra de Wacquant (2004), difiere en su énfasis sobre la alteración que la reeducación produce en todo patrón de interacción fuera de la prisión (o para el caso de Wacquant, el ring de boxeo y el gimnasio).

14 De hecho, hay dos números completos de Body \& Society (en 2013 y 2014) dedicados a este tema.

15 Otros académicos de la misma generación, Tyson Smith (2014) y Michal Pagis (2010), han extendido el estudio de la adquisición de saberes corporales a través del diálogo con la sociología fenomenológica y la psicología social.

16 Sarah Delamont y Neil Stephens (2008) utilizaron la modalidad de corporalización del capoeira para ilustrar la continuidad en el tiempo de Brasil en el cuerpo del practicante, con el propósito de conceptualizarlo como un habitus diaspórico.

17 Nota del traductor: en inglés “tempography”, refiere a una descripción amplia del orden sociotemporal nativo.

18 Otro grupo de investigadores que también merecen ser señalados son los que han estudiado las intersecciones entre habitus, estatus y clase: Ryan Centner (2008), que empleó el concepto para explorar la producción del capital espacial para el fenómeno de la apropiación de espacios por trabajadores de empresas punto-com en San Francisco; Amy Hanser (2008), quien utilizó habitus para explicar cómo vendedores fueron capaces de recuperar la confianza de sus clientes en un 
contexto de transformación de los mercados a través de la reconstrucción de la dimensión de clase de la experiencia socialista pasada que compartían; y Rachel Sherman (2007, pp. 254-55), que estudió la interacción entre trabajadores e invitados en hoteles lujosos. Aunque Sherman se concentra más en los límites, usa habitus/disposiciones para explicar cómo los consumidores ricos se reconocen a sí mismos interpelados por el hotel como individuos que pertenecen y son de una posición de clase a través de los servicios de lujo que consumen.

19 No incluyo en esta discusión críticas como las de Olivier Mongin, quién siguiendo a Sartrerechaza la "sociología determinista de Bourdieu" por reducir constantemente los márgenes de libertad del sujeto (Mongin y Roman, 1998).

$\underline{20}$ Incluso antiguos discípulos como Claude Grignon participaron de esta crítica sosteniendo que el hecho de que el habitus funcionara como una caja negra le permitió a Bourdieu "explicar con una sola palabra, definitiva, por qué los grupos sociales, y los individuos que los constituyen, reproducen comportamientos idénticos” (Grignon, 1996, p. 96).

$\underline{21}$ Uno de los elementos más frustrantes de la lectura de Bourdieu es que, aunque él mismo afirma rechazar el establecimiento de un marco teórico general y resalta que son los conceptos los que deben ser puestos en práctica, gran parte de su trabajo está dedicado a discutir esos conceptos de un modo general (por ejemplo, en La razón práctica, Meditaciones Pascalianas, El Sentido Práctico, La Dominación Masculina, etc.).

22 Lahire (2011, p. 51) propone pensar en las disposiciones como condicionales, con el objetivo de reconstruir las disposiciones a través de la descripción de las prácticas, de reconstruir las circunstancias bajo las cuales son desplegadas y los elementos clave de la historia del actor.

$\underline{23}$ Lizardo (2004) se ha centrado en el origen cognitivo piagetiano de algunos de los conceptos fundamentales de su sociología; Lahire (2001, p. 147) se ha enfocado en la tensión entre el lenguaje cognitivo y las dinámicas que Bourdieu está interesado en describir y explicar.

$\underline{24}$ Steinmetz (2006, y 2013) ha remarcado el hecho de que en la obra de Bourdieu la combinación de las situaciones particulares que la agente confronta, con las disposiciones específicas que porta y los constreñimientos que el pasado y el colectivo ejercen en ella, puede ser encontrada bajo tres formas principales: integrado, inconexo y partido. La primera iteración de las múltiples modalidades aparece en La miseria del mundo (Bourdieu et al., 1999, p. 383), y la segunda en su Autoanálisis de un Sociólogo (2008a, pp. 99-101).

$\underline{25}$ En su libro (2001 [2011], p. 46) describe exactamente siete potenciales escenarios de desajuste, incluyendo dos que podrían pensarse también junto con el habitus escindido de Bourdieu y el efecto de histéresis.

26 Varios autores franceses (DeCertau, 1984; Rancière, 1987; Grignon y Passeron, 1989) ya han criticado este punto luego de la publicación de La Distinción.

$\underline{27}$ En el triple significado de la frase: continuar cronológicamente, reconocer la relación con su obra -Boltanski, por ejemplo, fue en sus comienzos parte de su grupo- y el intento de desafiarlo. 


\section{Referencias bibliográficas}

Abend, G.(2008). The meaning of 'theory'. Sociological Theory,26(2), 173-199. Alford, Robert \&Andras, Szanto (1996). Orpheus wounded: The experience of pain in the professional worlds of the piano. Theory and Society,25(1), 1-44.

Auyero, Javier (2012). Patients of the State. The Politics of Waiting in Argentina.Durham: Duke University Press.

Auyero, Javier \& Swistum,Deborah (2009). FlammableEnvironmental Suffering in an Argentine Shantytown. New York: Oxford University Press.

Benzecry, Claudio E. (2011). The Opera Fanatic: Ethnography of an Obsession. Chicago: University of Chicago Press.

Boltanski, L., (2012) [1990].Love and Justice as Competences - Three Essays on the Sociology of Action (translated by Catherine Porter). Cambridge (UK): Polity Press.

Boltanski, L \& Thévenot, L. (1999). The Sociology of Critical Capacity.European Journal of Social Theory,2(3), 359-377.

Boltanski,L.\& Thévenot, L.(2006) [1991].On Justification. The Economies of Worth. Princeton: Princeton University Press.

Boudon, R. (1998). Social mechanisms without black boxes.In P. Hedström \& R. Swedberg (Eds.).Social Mechanisms.An Analytical Approach to Social Theory (pp. 172-203). New York: Cambridge University Press.

Bourdieu, P. (1977). Outline of a Theory of Practice. Cambridge: Cambridge University Press.

Bourdieu, P._(1979). Algeria 1960: The Disenchantment of the World: The Sense of Honour: The Kabyle House or the World Reversed: Essays. Cambridge: Cambridge University Press.

Bourdieu, P. (1990). The Logic of Practice. Palo Alto: Stanford University Press.

Bourdieu, P. (1996). Rules of Art: Genesis and Structure of the Literary Field. Palo Alto: Stanford University Press.

Bourdieu, P. (1998). Practical Reason: On the Theory of Action. Palo Alto: Stanford University Press.

Bourdieu, P.(2000). Pascalian Meditations. London: Polity.

Bourdieu, P. (2008a). Sketch for Self Analysis.Chicago: University of Chicago Press.

Bourdieu, P. (2008b). Bachelor's Ball.The Crisis in Peasant Society in Bearne. Chicago: University of Chicago Press.

Bourdieu, P. (1999).The Weight of the World.Palo Alto: Stanford University Press. Bourdieu, P.\&Wacquant, L.(1992). An Invitation to Reflexive Sociology. Chicago: University of Chicago Press. Bourgois, P.\&Schonberg, J. (2007). Intimate apartheid: Ethnic dimensions of habitus among homeless heroin injectors. Ethnography,8(1),7-33. 
Bourgois, P.\&Schonberg, J. (2009). Righteous Dopefiend.Berkeley:University of California Press.

Brubaker. R. (1985). Rethinking Classical Theory: The Sociological Vision of Pierre Bourdieu. Theory and Society,14(6), 745-775.

Brubaker. R. (1993). InCraig Calhoun. E. LiPuma\& M. Postone (Eds.).Bourdieu: Critical Perspectives (pp. 212-234).Cambridge (UK): Polity Press; Chicago: University of Chicago Press.

Brubaker, R.\& Cooper, F. (2000). Beyond “Identity”.Theory and Society,29(1), 1-47.

Brubaker,R.;Loveman, M.\& Stamatov, P. (2004). Ethnicity as Cognition.Theory and Society,33(1), 31-64.

Camic, C. (1986). The Matter of Habit.American Journal of Sociology,91(5), 1039-1087.

Caputo, D. (2012). The yard face: The contributions of inmate interpersonal violence to the carceral habitus.Ethnography,14(2), 165-185.

Champagne, P.\& Christin, O.(2012). Pierre Bourdieu: une initiation. Lyon: Presses universitaires de Lyon.

Centner, R.(2008). Places of Privileged Consumption Practices: Spatial Capital, the Dot-Com Habitus, and San Francisco's Internet Boom.City \& Community,7(3), 193-223.

Corcuff, P. (2001). Le collectif au défi du singulier: en partant de l'habitus.In B. Lahire (Dir.).Le travail sociologique du Pierre Bourdieu: Dettes et critiques (pp. 95-120). Paris: La Découverte.

Corcuff, P. (2007). Les nouvelles sociologies. Entre le collectif et l'individuel.Paris: Armand Colin.

Crossley, N. (2014). Embodied Actors, Sociability and the Limits of Reflexivity.Body \&Society,20(2), 106-112.

DeCerteau, M. (1984). The Practice of Everyday Life. Berkeley: California University Press.

Decoteau, C. L. (2013a). Hybrid Habitus: Toward a Post-Colonial Theory of Practice.In J. Go (Ed.). Postcolonial Sociology. Political Power and Social Theory, Volume 24 (pp.263293). Emerald Group Publishing Limited.

Decoteau, C. L. (2013b). Ancestors and Antiretrovirals: The Biopolitics of HIV/AIDS in PostApartheid South Africa. Chicago: University of Chicago Press.

Delamont, S.\&Stephens, N. (2008). Up on the Roof. Cultural Sociology,2(1), 57- 75.Desmond, M. (2006). Becoming a firefighter. Ethnography,7(4), 387-421.

Delamont, S.\&Stephens, N. (2007). On the Fireline: Living and Dying with Wildland Firefighters. Chicago: University of Chicago Press.

DiMaggio, P. (1979). Review: On Pierre Bourdieu. American Journal of Sociology,84(6), 14601474.

DiMaggio, P.(1997). Culture and Cognition. Annual Review of Sociology, 23(1), 263-287.

Dosse, F. (1997). L'empire du sens. La humanisation des sciences humaines. Paris: La Découverte. 
Downey, G. (2008). Scaffolding Imitation in Capoeira: Physical Education and Enculturation in an Afro-Brazilian Art. American Anthropologist,110(2), 204-213.

Elias, N.(1993). Mozart: Portrait of a Genius. Berkeley: University of California Press.

Faure, S. (2000). Apprendre par corps. Socio-anthropologiehistorique du champ chorégraphique.Paris: La Dispute.

Foucault, M.(1977) [1969].What is an author?.InD. F Bouchard (Ed.).Language, Counter-Memory, Practice(pp. 124-127).New York: Cornell University Press.

Frére, B.(2004). Genetic Structuralism, Psychological Sociology andPragmatic Social Actor Theory Proposals for a Convergence of French Sociologies.Theory, Culture \& Society,21(3), 85-99.

Gorski, P. (2013). Bourdieu as a Theorist of Change.Bourdieu and Historical Analysis(pp. 1-15). Durham: Duke University Press.

Green, A. I. (2007). Erotic Habitus: Toward a Sociology of Desire. Theory and Society, 37 (6), 597626.

Green, A. I.(2008). The Social Organization of Desire: The Sexual Fields Approach.Sociological Theory. 26 (1), 25-50.

Grignon, C.\&Passeron, J. C. (1999). Le Savant et le Populaire. Misérabilisme et populisme en sociologie et en literature. Paris: Gallimard/Le Seuil.

Grignon, C.(1996). Le Savant et le lettré: oul'examend'unedésillusion. RevueEuropéenne des Sciences Sociales, 34(103), 81-98.

Gross, N.(2003). Richard Rorty's pragmatism: A case study in the sociology of ideas. Theory and Society, 32(1), 93-148.

Hage, G. (2013). Eavesdropping on Bourdieu’s philosophers.Thesis Eleven,114(1), 76-93.

Hancock, B. (2005). Steppin’ out of Whiteness. Ethnography, 6(4), 427-461.

Hancock, B. (2007). Learning How to Make Life Swing. Qualitative Sociology, 30(2), 113-133.

Hancock, B. (2013). American Allegory: Lindy Hop and the Racial Imagination.

Chicago: University of Chicago Press.

Hanser, A.(2008). Service Encounters. Class, Gender, and the Market for Social Distinction in Urban China.Palo Alto: Stanford University Press.

Hirschkind, C.(2001). The Ethics of Listening: Cassette-Sermon Audition in Contemporary Cairo. American Ethnologist, 28(3), 623-649.

Ignatow, G. (2009). Theories of embodied knowledge: new directions for cultural and cognitive sociology?.Journal for the Theory of Social Behaviour, 37(2), 115-135.

Khan, Shamus. 2011. Privilege: The Making of an Adolescent Elite at St. Paul's School.

Princeton: Princeton University Press. 
Lahire, B. (Ed.) (2001). Le travail sociologique du Pierre Bourdieu: Dettes et critiques.Paris: La Découverte.

Lahire, B. (2003). From the habitus to an individual heritage of dispositions. Towards a sociology at the level of the individual.Poetics, 31(4), 329-355.

Lahire, B. (2006). La Culture des Individus. Paris: La Découverte.

Lahire, B. (2008). The individual and the mixing of genres: Cultural dissonance and selfdistinction.Poetics, 36(2), 166-188.

Lahire, B. (2010a). The Double Life of Writers.New Literary History, 41(2), 443-465.

Lahire, B. (2010b). Franz Kafka. Eléments pour une Théorie de la Création Literaire. Paris: La Découverte.

Lahire, B. (2011). The Plural Actor. London: Polity.

Lamont, M.(2012). How Has Bourdieu Been Good to Think With? The Case of the United States. Sociological Forum, 27(1), 228-237.

Lareau, A. (2002). Invisible Inequality: Social Class and Childrearing in Black Families and White Families.American Sociological Review,67(5), 747-776.

Lareau, A. (2003). Unequal Childhoods. Class, Race, and Family Life. Berkeley: University of California Press.

Latour, B. (1993). We have never been modern. Cambridge: Harvard University Press.

Lizardo, O. (2004). The Cognitive Origins of Bourdieu's Habitus.Journal for the Theory of Social Behaviour, 34(4), 375-401.

Lizardo, O. (2009). Is a “special psychology” of practice possible? From values and attitudes to embodied dispositions. Theory \& Psychology, 19(6), 713-727.

Lizardo, O. (2012). The Three Phases of Bourdieu's U.S. Reception: Comment on Lamont.Sociological Forum, 27(1), 238-244.

Longest, K.; Hitlin, S. \&Vaisey, S. (2013). Position and Disposition: The Contextual Development of Human Values. Social Forces, 91(4), 1499-1528.

Mahler, M. (2008). The Day before Election Day. Ethnography, 12(2), 149-173.

Martin, J. L. (2011). The Explanation of Social Action. New York: Oxford University Press.

Mongin, O.\& Roman, J. (1998). Le populisme version Bourdieu ou la tentation du mépris. Esprit(1940-), 158-175.

O’Connor, E. (2005). Embodied Knowledge: Meaning and the Struggle Towards Proficiency in Glassblowing. Ethnography,6(2), 183-204.

O’Connor, E.. (2006).Glassblowing Tools: Extending the Body towards Practical Knowledge and Informing a Social World. Qualitative Sociology, 29(2), 177-193. 
O’Connor, E.. (2007). Hot Glass: The Calorific Imagination of Practice in Glassblowing.In C. Calhoun \&R. Sennett (Eds.).Practicing Culture(pp. 57-81). London: Routledge.

Pagis, M. (2010). From Abstract Concepts to Experiential Knowledge: Embodying Enlightenment in a Meditation Center. Qualitative Sociology,33(4), 469-489.

Rancière, J. (1987). The Ignorant Schoolmaster: five lessons in intellectual emancipation. Stanford: Stanford University Press.

Sallaz, J.(2010). Talking Race, Marketing Culture: The Racial Habitus In and Out of Apartheid. Social Problems,57(2), 294-314.

Scheer, M. (2012). Are Emotions a Kind of Practice (and Is That What Makes Them Have a History)? A Bourdieuian Approach to Understanding Emotion. History and Theory, 51(2), 193-220.

Smith, T. (2014). Fighting for Recognition: Identity, Masculinity, and the Act of Violence in Professional Wrestling. Durham: Duke University Press.

Sherman, R. (2007). Class Acts. Service and Inequality in Luxury Hotels. Berkeley: University of California Press.

Steinmetz, G. (2006). Bourdieu's disavowal of Lacan: Psychoanalytic theory and the concepts of “habitus” and “symbolic capital”. Constellations, 13(4), 445-464.

Steinmetz, G. (2013). Toward Socioanalysis: The "Traumatic Kernel” of Psychoanalysis and NeoBourdieusian Theory. In P. Gorski (Ed.).Bourdieu and Historical Analysis (pp. 108-130). Durham: Duke University Press.

Stevens, M.(2007). Creating a Class. College Admissions and the Education of Elites. Cambridge: Harvard University Press.

Turner, B. S. \& Wainwright, S. P.(2003). Corps de Ballet: the case of the injured ballet dancer. Sociology of Health \& Illness, 25(4), 269-288.

Vaisey, S. (2009). Motivation and Justification: A Dual-ProcessModel of Culture in Action.American Journal of Sociology, 114(6), 1675-1715.

Van den Berg, A. (1998). Is Sociological Theory Too Grand for Social Mechanisms?. In P. Hedström \& R. Swedberg (Eds.).Social Mechanisms: An Analytical Approach to Social Theory(pp. 204-237). Cambridge: Cambridge University Press.

Wacquant, L. (1993). Bourdieu in America: Notes on the Transatlantic Importation of Social Theory. In C. Calhoun; E. LiPuma, \& M. Postone.Bourdieu: Critical Perspectives (pp. 235-262). Chicago: University of Chicago Press.

Wacquant, L. (2004). Body and Soul: Notebooks of an Apprentice Boxer. New York: Oxford University Press.

Wacquant, L. (2011). Habitus as Topic and Tool: Reflections on Becoming a Prizefighter. Qualitative Research in Psychology, 8(1), 81-92. 
Wacquant, L. (2014a). Homines in extremis: what fighting scholars teach us about habitus. Body \& Society,20(2), 3-17.

Wacquant, L. (2014b). Putting Habitus in its Place: Rejoinder to the Symposium. Body \&Society,20(2), 118-139.

Wainwright, S.; Williams, C.\&Turner, B. S.(2006). Varieties of habitus and the embodiment of ballet.Qualitative Research, 6(4), 535-558.

Widick, R. (2003). Flesh and the Free Market: (On Taking Bourdieu to the Options Exchange). Theory and Society, 32(5/6), 679-723.

Winchester, D. (2008). Embodying the Faith: Religious Practice and the Making of a Muslim Moral Habitus. Social Forces, 86(4), 1753-1780.

Young, A. (1999). The (Non)Accumulation of Capital: Explicating the Relationship of Structure and Agency in the Lives of Poor Black Men. Sociological Theory,17(2), 201-227. 